\title{
A Study of Expression and Comparison of Passive Sentences in English, Chinese and Japanese
}

\author{
Zhao Yushan, Xu Lin, Li Lijun
}

School of Foreign Languages, North China Electric Power University, Beijing, China

\section{Email address:}

zhaoyushan1963@163.com (Zhao Yushan),863051586@qq.com (Xu Lin),11j0426@163.com (Li Lijun)

\section{To cite this article:}

Zhao Yushan, Xu Lin, Li Lijun. A Study of Expression and Comparison of Passive Sentences in English, Chinese and Japanese. Science Innovation. Vol. 4, No. 1, 2016, pp. 7-10. doi: 10.11648/j.si.20160401.12

Received: February 24, 2016; Accepted: March 15, 2016; Published: April 8, 2016

Abstract: Passive sentences are important in English, Chinese and Japanese, which also serve as one of the difficulties in language acquisition. Passive sentences consist of three components: object, agent and verb. This paper makes a comparison on English, Chinese and Japanese passive sentences from those three aspects and points out the similarities and differences among them, in the hope of providing reference for the study of these three languages.

Keywords: Passive Sentences, Object, Agent, Comparison

\section{被动句在英语、汉语和日语中的表达与对比研究}

赵玉闪, 许琳, 李丽君

华北电力大学外国语学院, 北京, 中国

\section{邮箱}

zhaoyushan1963@163.com（赵玉闪），863051586@qq.com（许琳），11j0426@163.com（李丽君）

摘要: 被动句在英汉日三种语言中都是重要句式, 也是语言习得中的难点之一。英汉日被动句由受事、施事、动词三 部分构成。本文从这三方面对英汉日被动句进行对比, 分析得出三种语言被动句的共性和个性特点, 以期为这三种语 言的学习提供参考。

关键词: 被动句, 受事, 施事, 对比

\section{1. 引言}

被动句是语言学习中的重点之一。对不同语言被动句 的对比分析有助于语言学习者更好地掌握这一重要句式。 近年来, 有关被动句对比的论文并不少见, 但多为两种语 言之间的对比。这些论文多从语法、语用、语义三个角度 进行对比分析。目前为止有关英汉日三种语言被动句对比 的文章较为零散。温穗君从结构特征、形成动因及语用功 能对比了中英日三种语言的被动句 [1]。但这种对比过于 笼统, 缺乏系统性。间俊宇在他的硕士论文中指出英汉日
三种语言被动句的特种, 并进行对比。但由于被动句的复 杂性, 该论文中所提到的汉语被动句仅限于又标志的被动 句 [2]。鉴于以上论文研究的不足, 本文从被动句的基本 组成部分入手展开对比。英、日、汉三种语言的被动句有 一个共同特点: 都由受事、施事和动词三部分组成。正是 这一共性奠定了对比的基础, 才使对比成为可能。本文分 别从这三方面对比英汉日三种语言的被动句。 


\section{2. 受事}

受事的分析将从受事性质、受事省略情况以及受事中 整体和部分是否可分三个方面展开探讨。

\section{1. 受事性质}

从受事的性质而言, 三种语言中的受事既可以是有情 的人也可以是无情的物。但是三者的侧重有所不同: 英语 为表客观性, 重物称, 受事为无情物的比重较高。汉语和 日语传统的用法里, 受事都为有情物, 两种语言在受到以 英语为代表的西方语言的影响, 受事为无情物的用法增多。 但是, 日语无情物为主语的情况比汉语少 [3]。因此英、 汉、日三语中, 无情物为主语的比重依次降低。英语被动 句受事中有一种特殊情况, 即It作形式主语的被动句。这 种情况在日语和汉语的被动句中根本不存在, 但两种语言 分别有自己的表达形式。

\section{2. 受事省略}

受事省略情况也各有特点。英语属于形合语言, 形式 完整才能表达完整的意义。另外, 主语在英语句子中的地 位好比人的头部, 其重要性不言而喻。作为主语的受事不 能省略。汉语重意合, 在一定的语境下若受事明确, 不影 响理解, 主语可以省略, 但并不常见。省略主语是日语语 言的一大特点, 尤其当主语为第一人称时, 一般都会省略。 因此可以看出, 从英语、汉语、日语三者的受事的缺省性 势逐渐增强。

\section{3. 受事中的整体和部分}

受事中整体和部分是否可分是被动句学习的重点和 难点。汉语中当受事中存在整体 (常为有情物) 和部分 (常 为有情物的所有物、身体部位或与其有关联者)的关系时, 二者具有可分离性。整体和部分可以同时位于谓语动词前, 作句子主语。也可分别位于谓语动词前后。

1a团长的腿让炮弹炸伤了。

$1 \mathrm{~b}$ 团长让炮弹炸伤了腿。

例 $1 \mathrm{a}$ 和 $1 \mathrm{~b}$ 中, 整体是有情物 “团长”, 是间接受事者; 部分为无情物 “腿”, 是直接受事者。此句中, 动词的直 接受事为 “腿” 而非 “团长”。需要指出的是, 汉语被动 句中的受事虽具有可分离性，但不分离的情况更为常见。

在日语被动句中, 整体和部分只能分离, 二者分别位 于施事前后, 同处于谓语动词前。因此日语一般采用 $2 \mathrm{~b}$ 的说法而非 $2 \mathrm{a}$ 。

2a弟の手は犬に噛まれた。（X)

$2 \mathrm{~b}$ 弟は犬に手を噛まれた。

英语则恰恰相反, 整体和部分只能一同充当被动句的 主语, 偶尔也会出现受事分离, 但具有很大的限制性, 需 满足两个条件: 动词后的宾语为人身体的一部分; 须用介 词连接 [4]。因此3b不符合英语语法。汉语和日语的受事 分离则无此限制。

3a His head was beaten.

$3 \mathrm{~b}$ He was beaten head. $(x)$

$3 c$ He was beaten on the head.
总而言之，当被动句的受事存在整体部分关系时，英 语被动句的受事一般不具有分离性。汉语可以分离, 但不 普遍。日语受事必须分离。英、汉、日分离性逐渐增强。

表1 英汉日被动句受事对比。

\begin{tabular}{|c|c|c|c|}
\hline 对比项 语种 & 英 & 汉 & 日 \\
\hline 无情物作主语 & 多 & 中 & 少 \\
\hline 形式主语 & 有 & / & / \\
\hline 缺省性 & 弱 & 中 & 强 \\
\hline 整体部分分离性 & 弱 & 中 & 强 \\
\hline
\end{tabular}

\section{3. 动词}

动词部分的对比主要从动词的性质和动词的形式两 方面展开。

\section{1. 动词性质}

从动词的性质而言, 大部分及物动词都有被动语态, 而少数不及物动词在加上相应的介词后可以变成及物结 构, 构成被动句。英语被动句的谓语动词还可以是转述类 动词。It is said/reported/alleged that... 英语中心 理动词也可以构成被动句。这类动词有move、surprise、 touch、annoy、amuse等等。

4a This was made in China. (及物动词)

$4 \mathrm{~b}$ His hat has been sat on. (不及物动词)

4c This university is said to be the best one in China. (转述类动词)

4d The audience were deeply touched by his words. (心理动词)

汉语中及物动词和少数不及物动词可以构成被动句。 不及物动词需加上表因果关系的 “得” 字补语方可构成被 动句。英语转述类动词做谓语动词的被动句在汉语中没有 相应的被动表达, 汉语习惯用主动语态来表达: 据说, 据 称等。汉语中的心理动词也可以进入被动句 [5]。吓、感 动、惊呆等等。

$5 \mathrm{a}$ 他被老师表扬了。（及物动词）

$5 \mathrm{~b}$ 妈妈被孩子哭得心烦意乱。(不及物动词)

$5 \mathrm{c}$ 据说这所大学是中国最好的大学。(转述类动词)

$5 \mathrm{~d}$ 听众被他的话深深地感动了。(心理动词)

$5 \mathrm{a}$ 中的及物动词“表扬”直接客观的作用于受事“他”。 这类被动句被称为直接被动句。5b主语 “妈妈” 并非不及 物动词 “哭” 的直接作用的对象, “妈妈” 是主观间接的 收到了影响。动作的受事 “孩子” 并没有想要影响 “妈妈” 的意图。动词后面还有 “得” 字补语来说明受影响的结果 和状态。这类被动句称为间接被动句。这类句子中暗含着 一种因果关系, 因为孩子哭, 所以妈妈心烦意乱。分析可 见, 汉语中不及物动词进入被动句限制较多, 因此这类被 动句为数不多。

日语的动词主要的分类是分为他动词和自动词。他动 词和及物动词类似, 自动词和不及物动词相仿, 但它们都 不完全等同。他动词构成的被动句中，由于主语直接客观 地受到他动词的影响, 是动词的直接作用对象, 因此被称 
为直接被动句。自动词构成的被动句一般为因果复句, 主 语并非直接客观受到自动词的影响, 而是间接主观的受到 了影响, 而且通常是消极负面的影响。施事也没有想要影 响受事的意愿 [6]。因此这类被动句被称为间接被动句。 与英、汉语相比而言, 日语中的间接被动句发达。日语在 明治维新之后, 受西方语言的影响出现了一种惯用被动句 式, 主要用于报道和陈述常识性内容如: “ と言われて いる/ とされている/ と思われている”等等 $[7]$ 。这恰 好和英语中的转述类动词相对应。日语中的类似于 “びっ くりする、感動する” 等心理动词不能构成被动句, 只能 用主动表达, 这点和英语、汉语不同。

$6 \mathrm{a}$ 張さんは社長に叱られた。(他动词)

$6 \mathrm{~b}$ 李さんは子供に泣かれて、困っている。（自动 词)

$6 \mathrm{c}$ この大学は中国で一番いい大学と言われていま す。(转述类动词)

$6 \mathrm{~d}$ 聴衆は彼の話に深く感動した。（心理动词）

6a中他动词 “叱られた” 直接客观地作用于受事 “張 d $h$ ”, 为直接被动句。6b是一个因果复句, 分句中的 “泣 加” 为自动词, 受事 “李さん” 并非动词的直接宾语, 而是主观间接的受到了自动词 “泣力れ” 的影响。句子的 后半段 “困っている”为主句, 说明影响后的结果。有时 候被动句也可单独出现, 比如: 昨日は雨に降られた。被 雨淋的结果是不言而喻的, 因此无需言明。

\section{2. 动词形式}

英汉日被动句的谓语动词不仅仅在性质上各有特点, 动词的形式也存在差异。英语是屈折语, 被动语态由助动 词be/get+动词过去分词。除少数不规则动词外, 英语动 词的过去分词一般由动词 $+\mathrm{ed}$ 构成。如例句 $7 \mathrm{a}$ 。日语为黏 着语, 也是通过动词的形态变化来传达被动意义。日语被 动语态由动词 +5 れ $/$ れる构成, 如例句 $7 \mathrm{~b}$ 。汉语是孤立 语, 被动句中的谓语动词一般不能是光杆动词 [7]。在英 语和日语中通过动词形态变化传达的意义在汉语中需要 补充相应动态助词 “了”、“着”、“过” , 如 $7 \mathrm{c}$ 。少数 光杆动词也可以构成被动句, 具体有两种情况, 一种是固 定词组: 被捕、被杀、被盗等; 另外一种是动词本身是动 结式动词: 包围、收买、消灭、提名等。

7a The team was upgraded to second place in the $4 \times 100$ relay final at 2015 World Athletics Championships.

$7 \mathrm{~b}$ 第15回世界陸上選手権大会は本年8月、北京で開 催されます

$7 \mathrm{c}$ 就这样被感动着。

表2 英汉日被动句动词对比。

\begin{tabular}{|c|c|c|c|c|}
\hline \multicolumn{2}{|c|}{ 对比项 语种 } & 英 & 汉 & 日 \\
\hline \multirow{4}{*}{$\begin{array}{l}\text { 性 } \\
\text { 质 }\end{array}$} & 及物/他动词 & 多 & 中 & 少 \\
\hline & 不及物/自动词 & 少 & 中 & 多 \\
\hline & 转述类动词 & 有 & 无 & 有 \\
\hline & 情感类动词 & 可用 & 可用 & 不可用 \\
\hline \multicolumn{2}{|c|}{ 形式 } & $\begin{array}{l}\text { 助动词+动词 } \\
\text { 过去分词 }\end{array}$ & $\begin{array}{l}\text { 动词+了、 } \\
\text { 过、着 }\end{array}$ & $\begin{array}{l}\text { 动词+被动助 } \\
\text { 动词 }\end{array}$ \\
\hline
\end{tabular}

\section{4. 施事}

施事部分主要从施事的性质和省略情况两方面进行 对比。

\section{1. 施事性质}

从施事的性质而言, 英、汉、日被动句的施事既可以 为有情物也可以为无情物, 但是各有侧重。英语注重客观 性, 无情物作施事的比重较大。汉语和日语多采用主观视 角, 无情物做被动句施事的情况不如英语普遍。

8a The history is made by people. (有情物施事)

$8 b$ The flowers were destroyed by the rain. (无 情物施事)

$9 \mathrm{a}$ 车子被小明骑走了。（有情物施事） 施事)

$9 \mathrm{~b}$ 夜空被五彩缤纷的焰火照的光彩夺目。（无情物 事)

10a 空海は皇帝から大いに称賛された。（有情物施

10b 空が黒い雲に覆われている。（无情物施事）

\section{2. 施事省略}

英汉日被动句施事部分的省略也各有特点。英语被动 句的施事前必须由介词by/with/of等引导, 位于谓语动词 之后。省略介词和施事是英语被动句的一大特点。据 Jesperson统计, 约有 $70 \%$ $80 \%$ 的英语被动句中施事不出现 [8]。Quirk也指出, 英语中约有 $4 / 5$ 的被动句没有提施动 者。英语被动句的介词和施事不具有分离性, 不可单独出 现或省略。如:

11a The president was shot by a man in a restaurant. (介词施事同时出现)

11b The president was shot in a restaurant.（介 词施事同时省略)

汉语被动句可分为两种: 有标被动句和无标被动句。 句子中出现 “被”、“叫”、“让”、“给”等介词的 被动句叫做有标被动句。由于有标被动句对动词限制相 对较多, 因此这类被动句只是汉语被动句中的一部分。 汉语动词本身没有被动语态, 主要通过语序其次才是介 词被来表示被动意义, 所以介词并非汉语表达被动意义 的必要条件。省略介词的无标被动句又称为受事主语句。 只要不影响句子受动关系, 汉语倾向于省略介词和施事, 用主动语态表达被动意义 [9]。因此受事主语句在汉语中 为数众多, 是汉语被动句的一大特点。

$12 \mathrm{a}$ 全世界都被此次阅兵所震撼。（介词施事同时出 现）

$12 \mathrm{~b}$ 他已派到国外当记者了。（介词施事同时省略）

$12 \mathrm{c}$ 自行车被骑走了。（施事省略）

$12 \mathrm{~d}$ 自行车妹妹骑走了。(介词省略)

日语被动句受事后必须加上格助词に、から、によっ て, 格助词相当于和英语和汉语中的介词。日语和英语一 样, 格助词和施事不可分离, 只能同时出现或同时省略。 但是日语省略施事的情况远没有英语普遍。 
13a山本さんは知らない人に殴られた。（施事和格 助词同时出现）

$13 b$ 今回の会議は北京で開かれる。（施事和格助词 同时省略）

表3 英汉日被动句施事对比。

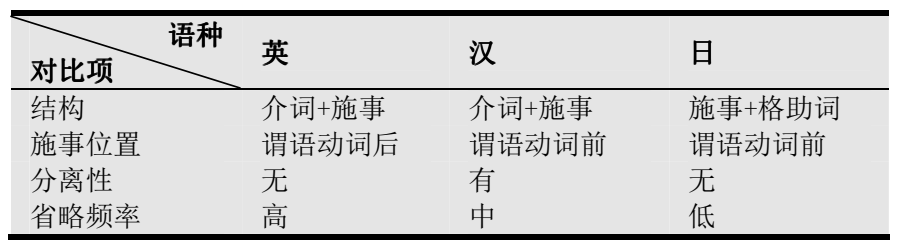

\section{5. 结束语}

本文从受事、施事、动词三方面对英汉日被动句进行 对比, 结果表明, 三种语言的被动句各有特点: 英语重形, 被动句在句法形态上体现为助动词后接动词过去分词。被 动句中多为无情物作受事并且省略施事; 汉语重意, 通过 语序其次是介词来表达被动意义, 用主动语态表被动意义 的受事主语句占据相当比重; 日语动词有专门的被动态, 最大特点是省略受事的间接被动句较为发达 [10]。整体而 言, 英语善用被动句, 被动句使用频率高, 汉语多用主动 句, 被动句使用频率低。而日语中的被动句没有英语那么 大行其道, 适用范围比汉语要广。

\section{致谢}

本研究由中央高校基本科研业务费专项资金资助 (华 北电力大学, 项目号：2014 ZD25）

\section{参考文献}

[1] 温穗君. 中英日被动句对比研究 [J]. 江西科技师范学院学 报, 2008, 03:73-76。

[2］刘畅. 英、日、汉被动句对比分析 [D]. 郑州大学, 2010。

[3] 徐否, 日语被动句学习难点解析 [J], 日语学习与研究, 2006, $03: 82-85$ 。

[4] 钟佩玲. 汉英被动句对比研究 [D]. 湖南大学, 2012。

[5] 段妞妞. 汉语儿童行为动词与心理动词被动句习得研究 [D]. 湖南大学, 2014。

[6] 杉村博文, 从日语的角度看汉语被动句的特点 $[\mathrm{J}]$, 语言文 字应用, 2003，02:64-75。

[7] 郭鸿杰, 韩红. 语料库驱动的英汉语言接触研究: 以 “被” 字 句为例 [J]. 外语教学与研究, 2012, 03:359-370+479.

[8] 雯燕萍. 英语被动语态的人际功能 [J]. 外语研 究, 2008, 02:22-26。

[9] 武咪咪. 概念迁移视角下英汉被动句的双向习得研究 [D]. 太原理工大学, 2015。

[10］杉村博文. 从日语的角度看汉语被动句的特点 [J]. 语言文 字应用, 2003, 02:64-75。 